\title{
Infantile urolithiasis
}

\author{
Maria Goretti Moreira Guimarães Penido ${ }^{1,2}$ - Uri Saggie Alon ${ }^{3}$
}

Received: 30 October 2020 / Accepted: 1 December 2020 / Published online: 2 January 2021

(C) IPNA 2021

\section{Introduction}

Pediatric urolithiasis has become an important worldwide medical concern as its incidence and prevalence have increased exponentially in both adults and children $[1,2]$. Its true incidence in childhood may be higher than has previously been observed, and 9-23\% of the total pediatric urolithiasis cases may present already during the first year of life [3, 4].

Once a stone disease is diagnosed, all efforts should be made to identify its etiology, in order to apply nonpharmacological and as necessary pharmacological intervention to minimize the risk of formation of additional stones. The etiology of urolithiasis is multifactorial, and in general can be divided between genetic and acquired causes. The latter group includes several categories, among them dietary, lifestyle, environmental, infectious, anatomical, and drug-related disorders. A significant number of patients may have more than one risk factor, while on the other hand, no cause can be found in a few patients [5-7]. Whereas in adults the leading causes of stone formation are relative oliguria, hypercalciuria, hypocitraturia, hyperuricosuria (or changes in urine acidity resulting in uric acid crystallization), and absorptive hyperoxaluria, causes in school-aged children in the Western world include mostly relative oliguria, hypercalciuria, and hypocitraturia $[1,2,8]$. In other parts of the world, and especially where consanguinity is still common, a high incidence of autosomal recessive disorders like oxaluria and cystinuria can be seen. In other places infectious etiologies, especially when superimposed on anatomic abnormalities, are a common cause of stone formation $[3,7]$.

Uri Saggie Alon

ualon@cmh.edu

1 Pediatric Nephrology Unit, Nephrology Center, Santa Casa de Belo Horizonte Hospital, Belo Horizonte, Minas Gerais, Brazil

2 Pediatric Nephrology Unit, Clinics Hospital, School of Medicine, Federal University of Minas Gerais, Belo Horizonte, Minas Gerais, Brazil

3 Bone and Mineral Disorders Clinic, Division of Pediatric Nephrology, Children's Mercy Hospital and Clinics, University of Missouri at Kansas City, Kansas City, MO, USA
Data on urolithiasis in newborns and infants are scarce. While extremely preterm infants are at high risk for stone formation, often secondary to the use of furosemide for treatment of their lung condition, its etiology in full-term infants is yet not well understood [7, 9]. According to Alpay et al. [3] and Serdaroğlu et al. [7], infantile urolithiasis appears to be a separate clinical entity in terms of both the etiological characteristics and the clinical course of the disease. Nevertheless, also among this population, hypercalciuria has been reported as the most frequent metabolic abnormality [3, 5, 10, 11]. Additional etiologies of urolithiasis in infancy include hypocitraturia, hyperoxaluria, hypomagnesuria, systemic diseases, urinary tract anatomic abnormalities and infections, and medications [3, 5, 12-14]. Besides genetic etiologies, one of the more important contributors to the changes in urine biochemistry leading to stone disease is dietary habits $[12,15$, 16]. Within this category of nutrition, a question commonly asked is the role of vitamin $\mathrm{D}$, which involves both exposure to sunlight and its supplementation, which could result in hypercalciuria $[12,15,17]$. Once infantile urolithiasis has been diagnosed, the next question asked regards its prognosis, including the development of new stones vs. complete recovery $[5,7,11,13]$.

In view of these questions, we were pleased to see the study by Yilmaz et al. published in this edition of Pediatric Nephrology [18]. The authors carried out a prospective cohort and case-control study on 30 infants with urolithiasis and 30 healthy control infants, all 60 being exclusively breastfed and all receiving daily standard supplementation with oral vitamin D. Data were collected about the season of the year in which the babies were born. Maternal milk was analyzed for stone formation promoters and inhibitors. Infants' serum was analyzed for kidney function, electrolytes, minerals, 25(OH)-vitamin $\mathrm{D}$, and parathyroid hormone (PTH), and their random urine samples were studied for stone formation promoters and inhibitors, and urine calcium/citrate was calculated. All samples were then compared between patients and controls. Longterm follow-up of the infants with urolithiasis with periodic ultrasonography allowed the authors to study the natural history of the stone disease in their patients. 


\section{Results of Yilmaz et al. study}

The authors used relatively stringent criteria to identify the participants and thus minimize possible errors in the data collection and analysis. A previous study assessing serum $25(\mathrm{OH})$-vitamin D levels in infants was used as reference to calculate the power analysis which was met by studying 30 infants in each group [14]. Inclusion criteria dictated that, at the start of the study, all participants be at 1 to 6 months age range, their birth weight $>2500 \mathrm{~g}$, have normal anthropometric data, solely being breastfed supplemented by oral vitamin D (400 IU/day), have normal kidney function, do not use any drugs, and have no systemic disease or history of urinary tract infection. Infants with anatomic abnormalities that may predispose to stone formation were excluded. In both groups, maternal milk was analyzed for calcium, oxalate, uric acid, phosphate, citrate, and magnesium. The infants' blood samples were assessed for creatinine, urea, sodium, potassium, chloride, bicarbonate, uric acid, calcium, phosphate, magnesium, venous blood gases, 25(OH)-vitamin D, and PTH. Random urine analysis included the evaluation of calcium, cystine, uric acid, oxalate, magnesium, and citrate, all expressed as their ratio to creatinine, and sodium/potassium and calcium/citrate ratios were calculated. Patients were followed periodically clinically and by ultrasound. Statistical tools used, as detailed in the article, were appropriate. There was no difference between the study group and the control one concerning age, gender, male/female ratio, or family history of urolithiasis; however, significantly more infants with stone disease were born during the summer months. Biochemically, estimated glomerular filtration rate, serum sodium, potassium, chloride, magnesium, uric acid, and bicarbonate were similar in both groups. In contrast, mean serum calcium, phosphorus, and 25(OH)-vitamin D levels were significantly higher in patients at a time their mean serum PTH level was significantly lower compared with controls. Whereas all other parameters were identical in both groups, in infants with urolithiasis, urine calcium/creatinine and calcium/citrate ratios were significantly higher than in controls. The mean follow-up lasted 56 months during which time no new stones were reported. At last follow-up, the stones had spontaneously resolved in 25 of 27 remaining patients. The authors concluded that breast milk per se is not responsible for development of stones in infants, but supplementation with oral vitamin $\mathrm{D}$, particularly during the summer months, might be. The prognosis in this group of patients can be regarded as exceptionally good.

\section{Causes and prognosis of infantile urolithiasis}

Newborn babies and infants are a special group in relation to formation of urinary tract calcifications $[3,11,12]$. The calcifications can present as nephrocalcinosis, urolithiasis, or a combination of the two $[9,13]$. The risk factors for development of renal calcifications include short gestation, low birth weight, parenteral nutrition and possibly certain other types of artificial nutrition, use of medications like furosemide, glucocorticoids, and nephrotoxic medications, a prolonged oxygen supplementation or ventilation, presence of anatomic abnormalities of the urinary tract, systemic diseases and urinary tract infections $[9,13,19]$. In their study, Yilmaz et al. [18] were careful to exclude all the above etiologies by including well-defined groups of patients and matching controls. This allowed them to focus on the study's two main objectives, to identify the etiology of stone formation in breast-fed infants and explore the outcome of urolithiasis developed in this population.

The composition of the patient's diet is one of the more important risk factors for pediatric and adult urolithiasis $[8$, $20,21]$. The nutrients involved in the development of kidney stones are many, including sodium, oxalate, calcium, phosphate, magnesium, potassium, animal protein, sugars, citrate, and possibly others [20]. Consequently, whenever applicable, dietary interventions are used to combat stone formation. Indeed, implementation of the Dietary Approach to Stop Hypertension (DASH) diet has been shown to be beneficial in preventing urolithiasis [21]. A similar approach of decreased salt intake and increased potassium intake to decrease urine calcium excretion and stone formation, was demonstrated in the pediatric population [8]. Not surprisingly, Yilmaz and co-authors found no fault in breast milk, as shown also to be the case by others $[12,15,16]$.

Analyzing the urine, the authors found that when compared with the healthy controls, their stone forming infants had significantly higher calcium/creatinine and calcium/citrate values. Concomitantly, the stone forming infants were found to have significantly higher, albeit in many cases still within the normal range, serum levels of $25(\mathrm{OH})$-vitamin $\mathrm{D}$, calcium, and phosphate at a time PTH was lower, compared with controls. Although neither serum albumin nor ionized calcium concentrations were measured to assess filterable calcium, one can assume that more calcium absorbed from the gut was filtered in the glomeruli, and in the face of reduced PTH, less of it being reabsorbed in the tubules, thus resulting in hypercalciuria. As such, Yilmaz et al. concluded that it was the combination of supplementation with vitamin $\mathrm{D}$ and the time of the year the babies were born, namely, increased exposure to sunlight during the summer, which led to the development of hypercalciuria. As for the effect of sunlight, one cannot tell from this study whether the increased exposure to it was of mothers, their babies, or both.

The association between dietary vitamin D intake, serum 25(OH)-vitamin D level, and hypercalciuria was already observed by others, in research animals and human adults [22, 23]. A similar observation was made also in infants $[12,14]$. 
Fallahzadeh et al. [14] found serum levels of 25(OH)-vitamin $\mathrm{D}$ to be significantly higher in the infants with urolithiasis than in the controls, as well as positive correlation between serum levels of $25(\mathrm{OH})$-vitamin D and serum calcium. Milart et al. [24], studying children with hypercalciuria, observed a rise in serum 25(OH)-vitamin D following supplementation with vitamin $\mathrm{D}$ but no increase in calcium excretion. An extensive literature search concluded that the effect of oral vitamin D on urine calcium may vary between populations [23].

Although not one of the study's objectives, and as such not directly addressed by the authors, their findings bring up another issue of importance to students of pediatric urolithiasis. Evidently, though still higher than in controls, most if not all stone formers had their random urine calcium/creatinine ratio well within the normal range for age, thus questioning if this parameter can be used to identify the individual infant to be at risk for stone formation. What did seem to be a better indicator of risk for stone formation was urine calcium/citrate ratio which in most infants exceeded the upper limit of normal value of 0.33 established by Srivastava et al. [25] and DeFoor et al. [26]. Although in both studies the pediatric population investigated was older, urine calcium/citrate ratio was shown to be a more sensitive tool than calcium/creatinine ratio in assessing the risk for stone formation. This is possibly due to the fact that it incorporates the most common stone promoter and inhibitor, while excluding the muscle mass-dependent creatinine [25, 27]. As such, it will be important to establish normative urine calcium/citrate data in infants. It will be further interesting to learn the effect of diet, and in particular breastmilk vs. formula, on this ratio.

Long-term follow-up, for up to 4.5 years, allowed the authors to observe that their patients did not develop new stones; no intervention, medical or surgical, was required; and the stones resolved spontaneously in the vast majority of the patients. A similar experience of good outcome was also reported by Naseri [11] who observed resolution of infantile urolithiasis with no need for intervention. Huynh et al. [13] followed 72 infants with nephrocalcinosis/urolithiasis and observed resolution of the calcifications in $73 \%$ of cases during a mean follow-up of 12 months, but some of their patients received pharmacological therapy. Downing et al. [22] reported that once the offending agent is removed, in their case furosemide, stone formation ceases. It is nevertheless important to point out that other studies found stone recurrence and a need for surgical intervention in some of the infants, especially when stones exceeded $5 \mathrm{~mm}$ in size $[5,12,28]$.

\section{Should all infants receive vitamin D supplementation during the summer months?}

There are three main sources of vitamin D, namely, nutrition, exposure to sunlight, and supplemental vitamin D. The content of vitamin D in breast milk is often too low to address the infant's need. Therefore, to obtain enough of the vitamin, babies must be exposed to sunlight properly and/or receive supplementation with vitamin D. As exposure to effective sunlight may vary by geographic location, ethnicity, cultural habits, and individual factors, the World Health Organization recommends daily supplementation with oral vitamin $\mathrm{D}$ at $400 \mathrm{IU} /$ day to all infants bellow age of 12 months, as was followed by the families of all study participants. Considering its level in controls, the higher serum $25(\mathrm{OH})$ vitamin D levels observed in the study's stone formers cannot be related to either nutrition or vitamin D supplementation per se. However, when it comes to the third contributor of vitamin D, sunlight, the authors observed that a significant majority of the babies with urolithiasis were born in the summer, while the majority of controls were born in the winter. A similar observation was made by Naseri [11]. An extensive review of previous studies documents the effect of the time of the year on endogenous vitamin D production, reaching its peak during the summer [17]. Thus, in this respect, the observation by Yilmaz et al. is confirmatory. As the study did not attempt to measure and compare breast milk's vitamin D content, we cannot tell to what extent it was maternal vs. infant's exposure to sunlight, which played a role in raising serum $25(\mathrm{OH})$-vitamin D level. In any event, the study's findings bring up the question of whether supplementation with oral vitamin $\mathrm{D}$ to infants should be universally mandatory everywhere and all times around the globe [14, 29]. The current study cannot shed more light on the question, as no longitudinal data were collected, namely whether serum $25(\mathrm{OH})$-vitamin D level and hypercalciuria in stone formers normalized during the winter and/or after discontinuation of supplementation with vitamin D. Future studies will have to address this intriguing matter.

\section{Conclusions}

The article by Yilmaz et al. improves the base of our knowledge and potentially our clinical practice. It has three main messages: (a) when it comes to urolithiasis, breast milk is at no fault, (b) the prognosis of urolithiasis in this specific population of breastfed infants is very good without a need for any intervention. However, while they portray a very rosy picture, other studies found that some infants do need either medical or surgical intervention; hence, good practice will be to continue to follow this population periodically and treat each case individually; (c) it is possible that the stone formation in the study's location is secondary to hypercalciuria which may be due to supplementation with vitamin D during the summer months. The latter observation invites the question of whether all breast-fed infants, with no exclusion, throughout the world and during all seasons of the year, should be supplemented with vitamin $\mathrm{D}$, or should a more pinpointed approach be adopted. Hopefully future studies will address these intriguing questions. 


\section{Compliance with ethical standards}

Conflict of interest The authors declare that they have no conflicts of interest.

\section{References}

1. VanDervoort K, Wiesen J, Frank R, Vento S, Crosby V, Chandra M, Trachtman H (2007) Urolithiasis in pediatric patients: a single center study of incidence, clinical presentation and outcome. J Urol 177:2300-2305

2. Penido MGMG, Srivastava T, Alon US (2013) Pediatric primary urolithiasis: 12-year experience at a Midwestern Children's Hospital. J Urol 189:1493-1497

3. Alpay H, Gokce I, Özen A, Bıyıklı N (2013) Urinary stone disease in the first year of life: is it dangerous? Pediatr Surg Int 29:311-316

4. Ali SH, Rifat UN (2005) Etiological and clinical patterns of childhood urolithiasis in Iraq. Pediatr Nephrol 20:1453-1457

5. Güven AG, Koyun M, Baysal YE, Akman SA, Alimoglu E, Akbas H, Kabaalioglu A (2010) Urolithiasis in the first year of life. Pediatr Nephrol 25:129-134

6. Milliner DS, Murphy ME (1993) Urolithiasis in pediatric patients. Mayo Clin Proc 68:241-248

7. Serdaroğlu E, Aydoğan M, Özdemir K, Bak M (2017) Incidence and causes of urolithiasis in children between 0-2 years. Minerva Urol Nefrol 69:181-188

8. Alon US, Zimmerman H, Alon M (2004) Evaluation and treatment of pediatric idiopathic urolithiasis-revisited. Pediatr Nephrol 19: 516-520

9. Downing GJ, Egelhoff JC, Daily DK, Alon U (1991) Furosemiderelated renal calcification in the premature infant. A longitudinal study. Pediatr Radiol 21:718-723

10. Demir F, Yavuz S, Kiyak A, Aydoğan G, Korkmaz O, Sarı F (2014) Infantile urolithiasis: a single center experience. IKSST Derg 6:137-141

11. Naseri M (2015) Urolithiasis in the first 2 months of life. Iran J Kidney Dis 9:379-385

12. Bastuğ F, Gündüz Z, Tülpar S, Poyrazoflu H, Düoünsel R (2013) Urolithiasis in infants: evaluation of risk factors. World J Urol 31: $1117-1122$

13. Huynh M, Clark R, Li J, Filler G, Dave S (2017) A case control analysis investigating risk factors and outcomes for nephrocalcinosis and renal calculi in neonates. J Pediatr Urol 13: 356.e1-356.e5

14. Fallahzadeh MH, Zare J, Al-Hashemi GH, Derakhshan A, Basiratnia M, Arasteh MM, Fallahzadeh MA, Fallahzadeh MK (2012) Elevated serum levels of vitamin $d$ in infants with urolithiasis. Iran J Kidney Dis 6:186-191

15. Hoppe B, Roth B, Bauerfed C, Langman CB (1998) Oxalate, citrate, and sulfate concentration in human milk compared with formula preparations: influence on urinary anion excretion. $\mathrm{J}$ Pediatr Gastroenterol Nutr 27:383-386

16. Campfield T, Braden G, Flynn-Valone P, Clark N (1994) Urinary oxalate excretion in premature infants: effect of human milk versus formula feeding. Pediatrics 94:674-678

17. Holick MF (2020) Sunlight, UV radiation, vitamin d, and skin cancer: how much sunlight do we need? In: Reichrath J (ed) Sunlight, vitamin D and skin cancer. Advances in Experimental Medicine and Biology, vol 1268. Springer, Cham. https://doi.org/ 10.1007/978-3-030-46227-7 2

18. Yilmaz N, Yuksel S, Altintas F, Kocygit A (2020) Nephrolithiasis during the first six months of life in exclusively breastfed infants. Pediatr Nephrol. https://doi.org/10.1007/s00467-020-04815-w

19. Narendra A, White M, Rolton H, Alloub Z, Wilkinson G, McColl JH, Beattie J (2001) Nephrocalcinosis in preterm babies. Arch Dis Child Fetal Neonatal Ed 85:F207-F213

20. Shin S, Srivastava A, Alli NA, Bandyopadhyay BC (2018) Confounding risk factors and preventative measures driving nephrolithiasis global make-up. World J Nephrol 7:129-142

21. Taylor EN, Fung TT, Curhan GC (2009) DASH-style diet associates with reduced risk for kidney stones. J Am Soc Nephrol 20: 2253-2259

22. Alon U, Wellons MD, Chan JCM (1983) Reversal of vitamin- $\mathrm{D}_{2-}$ induced hypercalciuria by chlorothiazide. Pediatr Res 17:117-119

23. Letavernier E, Daudon M (2018) Vitamin D, hypercalciuria and kidney stones. Nutrients 10:366-379

24. Milart J, Lewicka A, Jobs K, Wawrzyniak A, Majder-Łopatka M, Kalicki B (2020) Effect of vitamin D treatment on dynamics of stones formation in the urinary tract and bone density in children with idiopathic hypercalciuria. Nutrients 12:2521. https://doi.org/ 10.3390/nu12092521

25. Srivastava T, Winston MJ, Auron A, Alon US (2009) Urine calcium/citrate ratio in children with hypercalciuric stones. Pediatr Res 66:85-90

26. DeFoor W, Minevich E, Jackson E, Asplin J (2008) Urinary metabolic abnormalities in solitary and recurrent stone forming children. J Urol 179:2369-2372

27. DeFoor W, Jackson E, Schulte M, Alam Z, Asplin J (2017) Calcium-to-creatinine ratio distinguishes solitary and recurrent urinary stone forming children. Pediatr Urol 198:416-421

28. Saygili SK, Kirli EA, Tasdemir E, Canpolat N, Caliskan S, Sever L, Talat Z, Önal B (2020) Natural history of patients with infantile nephrolithiasis: what are the predictors of surgical intervention? Pediatr Nephrol. https://doi.org/10.1007/s00467-202-04781-3

29. Siafarikas A, Piazena H, Feister U, Bulsara MK, Meffert H, Hesse V (2011) Randomised controlled trial analysing supplementation with 250 versus 500 units of vitamin D3, sun exposure and surrounding factors in breastfed infants. Arch Dis Child 96:91-95

Publisher's note Springer Nature remains neutral with regard to jurisdictional claims in published maps and institutional affiliations. 